\title{
A new bioluminescent species of Mycena sect. Exornatae from Kerala State, India
}

\author{
Aravindakshan DM ${ }^{1}$, Kumar $\mathrm{TKA}^{2}$ and Manimohan $\mathbf{P}^{1^{*}}$ \\ ${ }^{1}$ Department of Botany, University of Calicut, Kerala, 673 635, India \\ ${ }^{2}$ Department of Botany, The Zamorin's Guruvayurappan College, Calicut, Kerala, 673 014, India
}

Aravindakshan DM, Kumar TKA, Manimohan P 2012 - A new bioluminescent species of Mycena sect. Exornatae from Kerala State, India. Mycosphere 3(5), 556-561, Doi 10.5943 /mycosphere/3/5/4

Recent studies on the genus Mycena in Kerala State, India resulted in the discovery of a new species, herein described as M. deeptha. It has hallmarks of Mycena section Exornatae such as gelatinous pileipellis composed of hyphae that are covered with thorn-like excrescences, nongelatinized stipitipellis, discoid stipe base and luminescent mycelium. A combination of characters such as larger basidiomata, fimbriate pileal margin composed of cells with elongate protrusion, cheilocystidia and caulocystidia with elongate apical projections, and the presence of detersile elements over the primordium, however, makes it unique within that section. Phylogenetic analyses based on ITS rDNA sequences using maximum parsimony and maximum likelihood methods support $M$. deeptha as a distinct species belonging to a clade containing $M$. chlorophos, a wellknown luminescent species of sect. Exornatae. The ITS rDNA sequence of $M$. deeptha shows 99$100 \%$ similarity with those of two unidentified Mycena species (environmental samples) deposited in GenBank indicating that the new species may have a wider geographical distribution.

Key words - Agaricales - Basidiomycota - biodiversity - molecular phylogeny - Mycenaceae

\section{Article Information}

Received 20 August 2012

Accepted 27 August 2012

Published online 12 September 2012

*Corresponding author: P Manimohan - e-mail - pmanimohan@gmail.com

\section{Introduction}

Several Mycena (Mycenaceae, Agaricales, Basidiomycota) species are renowned for their ability to emit visible light. Eighty three species of luminous fungi are currently known (Vydryakova et al. 2011), of which 35 species belong to the genus Mycena, distributed in 17 sections (Desjardin et al. 2008). In many species, only the mycelium is luminescent, whereas in others both the mycelium and the basidiomata emit light. In a few species, only the basidiomata have been reported as luminescent while the mycelium is nonluminescent $(M$. irritans $\mathrm{E}$. Horak, $M$. lamprospora (Corner) E. Horak, M. sublucens Corner) (Corner 1954, Horak 1978, Desjardin et al. 2007, 2010). In M. polygramma (Bull.) Gray and in $M$. lamprospora spores are luminescent (Herring 1994, Horak 1978). While some species such as M. chlorophos (Berk. \& M.A. Curtis) Sacc. are brightly luminescent, some like M. haematopus (Pers.) P. Kumm., show only low-level bioluminescence, detected only by using a photometer.

During our studies on the genus Mycena in Kerala State, India, we discovered a species whose basidiomata were non-luminescent, but mycelium, both on its natural substratum and in culture, exhibited bioluminescence. Conventional morphology-based observations and ITS sequence-based phylogenetic analysis revealed it to be a new species, which is described, 
illustrated and discussed here.

\section{Methods}

\section{Morphological studies}

Microscopic observations were made on material stained with $1 \%$ aqueous solution of Congo red and mounted in $3 \%$ aqueous $\mathrm{KOH}$. Melzer's reagent was used to observe whether the spores and tissues were amyloid or vinoid. For evaluation of the range of spore size, twenty basidiospores from each specimen cited were measured. Mycelium of the agaric was cultured on malt extract agar by transferring basidiospores from spore prints to sterilized medium under aseptic conditions. Photographs of the bioluminescent mycelial culture were taken using a digital camera in a dark room with 40-50 minutes of exposure. Colour codes used in the description are from Kornerup \& Wanscher (1978). The examined collections are deposited at Kew (Mycology) Herbarium and the Kew accession numbers (e.g., K (M) 178331) are indicated. The infrageneric taxonomy of Mycena followed is that of Maas Geesteranus (1992).

\section{DNA extraction and sequencing}

DNA extractions were made from freshly collected basidiomata using a commercial DNA extraction kit (Sigma GenElute $^{\mathrm{TM}}$ Plant Genomic DNA Miniprep kit, Sigma-Aldrich Corp., St. Louis). PCR reactions were performed with primers ITS1 and ITS5. Amplification reactions were performed in MJMini TM thermal cycler (BioRad, Hercules). Amplified PCR product was purified using column purification (GenEluteTM PCR Clean-Up kit, SigmaAldrich Corp., St. Louis) as per manufacturer's guidelines and was subjected to automated DNA sequencing on ABI3730xl Genetic Analyzer (Applied Biosystems, USA) using primers ITS1 and ITS5. The generated sequence was edited manually using BioEdit sequence alignment editor version 7.0.9.0 (Tom Hall, Ibis Biosciences, Carlsbad, California). The edited sequence was then used for BLAST searches in the GenBank database (www.ncbi.nlm.nih.gov). The newly generated sequence was deposited in GenBank (accession number JX481737).

\section{Sequence alignment and phylogenetic analysis}

The newly generated ITS sequence (742 bp) and those retrieved from GenBank (21 ITS sequences) were initially aligned using CLUSTAL X 2.1 and then checked in Se-Al 2.0a11 (Andrew Rambaut, University of Edinburgh, U.K.; http://tree.bio.ed.ac.uk/software/seal). The sequences from GenBank were selected based on their similarity indices; available sequences of representative species of related sections of Mycena were also added to the dataset. Tricholoma aestuans (Fr.) Gillet and Suillus pictus (Peck) A.H. Sm. \& Thiers were selected as outgroup taxa for rooting purposes. Maximum Parsimony analysis was conducted using PAUP* 4.0b10 (Swofford 2002) using heuristic search option with tree bisection-reconnection (TBR) branch swapping algorithm, with 20 replicates using random step-wise addition and holding one tree at each step. All minimal length trees were saved and maxtrees were set as unrestricted. Bootstrap analysis (Felsenstein 1985) was carried out with full heuristic search option on 1000 replicates. Bayesian analysis was done using MrBayes 3.1.2 (Ronquist \& Huelsenbeck 2003). The general time reversible model with gamma distributed rate variation option was selected for the analysis. Multiple independent analyses were done for 2 million generations, with trees saved every 100 generations. Options for incremental heating scheme, chain numbers etc. were set to MrBayes default settings. The aligned sequence data matrix has been deposited in TreeBase (http://purl.org/phylo/treebase/phylows/study/T B2:S13309).

\section{Results}

\section{Taxonomy}

Mycena deeptha Aravind. \& Manim. sp. nov. MycoBank MB 801152

Figs 1-11

Etymology - deeptha (Sanskrit), luminous.

Characterised by a gelatinous pileipellis composed of hyphae that are covered with thorn-like excrescences, non-gelatinized stipitipellis, discoid stipe base and a luminescent 


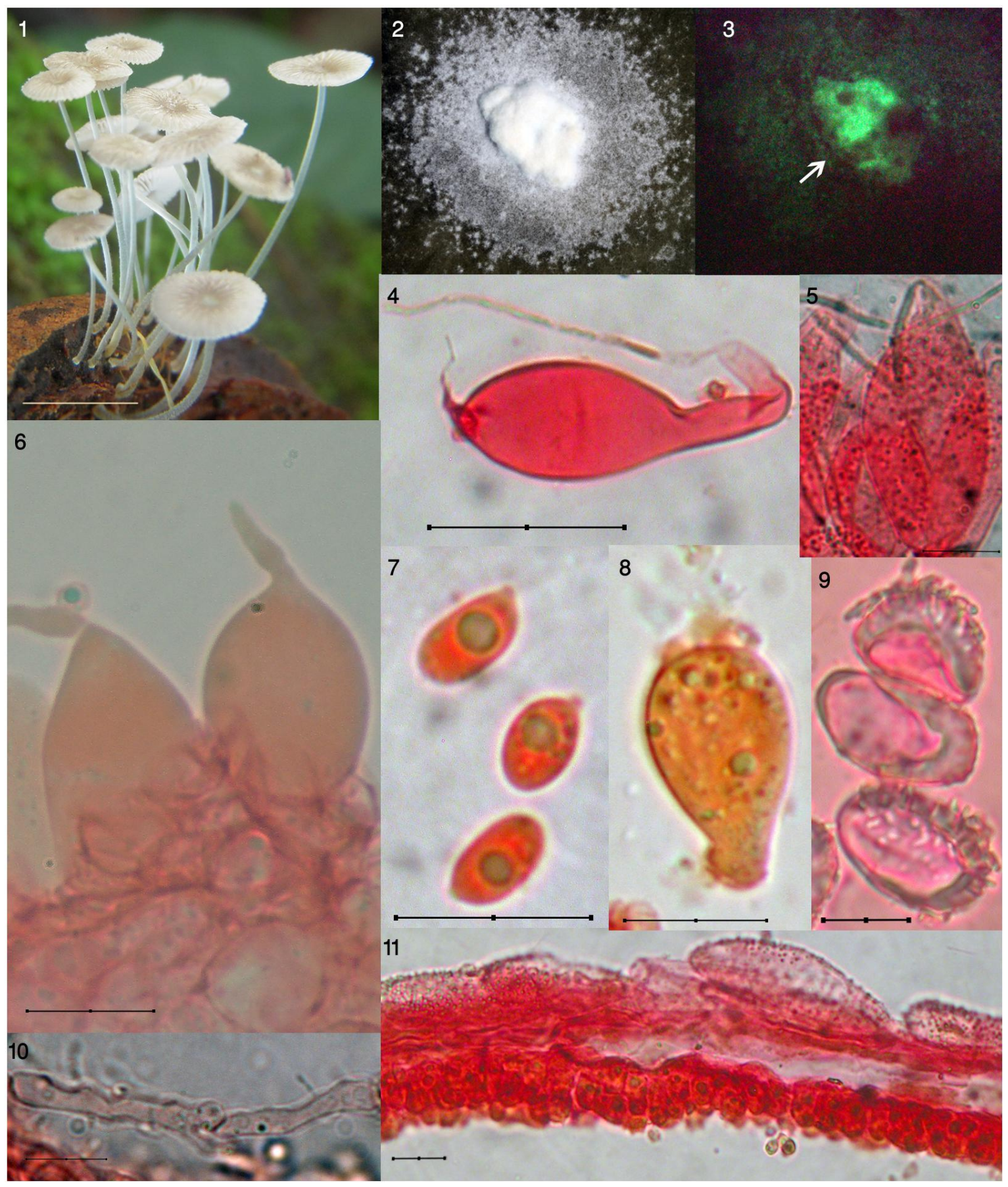

Figs 1-11 - Mycena deeptha. 1 Basidiomata. 2 Cultured mycelium on agar medium. 3 Same mycelium in darkness showing bioluminescence. 4 Caulocystidium. 5 Pileus marginal cells. 6 Cheilocystidia. 7 Basidiospores. 8 Basidium. 9 Elements over the primordium. 10 Hypha of the pileipellis with thorn-like excrescences. 11 Radial section of the pileus showing structure of the pileipellis - Bars $=10 \mathrm{~mm}$ for basidiomata and $10 \mu \mathrm{m}$ for micro-structures.

mycelium. Differing from other known members of the section Exornatae such as Mycena chlorophos, $M$. discobasis Métrod, $M$. chlorinosma Singer and M. margarita (Murrill) Murrill owing to its combination of characters such as larger basidiomata, fimbriate pileal margin composed of cells with elongate protrusion, cheilocystidia and caulocystidia with elongate apical projections, and the presence of detersile elements over the primordium.

Basidiomata small, delicate. Pileus 1-9.5 $\mathrm{mm}$ diam., $0.5-0.75 \mathrm{~mm}$ high, parabolic to conic when young, becoming nearly applanate 
with a centrally flattened depression or rarely umbilicate with age; surface yellowish grey (4B2) or dark blonde (5D4) at the centre, tobacco brown $(5 \mathrm{~F} 6)$ in the middle, and whitish or yellowish white (4B1) towards margin when young, becoming brownish orange $(5 \mathrm{C} 3)$ at the central depression, greyish brown (5D3) in the middle and brownish grey (5C2) to marble white (5B2) towards margin with age, translucent-striate when young, becoming plicato-sulcate with age, finely pruinose all over, dry; margin initially straight, becoming plane or upturned, fimbriate. Lamellae 15-18(23) reaching the stipe, free or narrowly adnate and then seceding to form a pseudocollarium, pale greyish or whitish, thin, $0.25-0.5 \mathrm{~mm}$ wide, close, with lamellulae of 2-5 lengths; edge finely hairy under a lens, concolorous with the sides. Stipe $6-30 \times 0.25-0.5 \mathrm{~mm}$, central, terete, almost equal when very young, tapering towards apex with age, hollow; surface translucent, white to cream (4A3), becoming yellowish or greyish yellow (4B3) or yellowish grey (4B2), entirely densely pubescent when young, becoming almost glabrous towards apex with age; base discoid, of radiating mycelium, hairy. Context not conspicuous, less than $0.5 \mathrm{~mm}$ wide. Odour and taste not distinctive. Basidiomata not luminescent, but the mycelium (that on the natural substratum as well as that cultured on malt extract agar) shows faint greenish luminescence.

Basidiospores $6.5-9(-11) \times 3-5(-5.5)$ $(7.64 \pm 0.386 \times 3.868 \pm 0.162) \mu \mathrm{m}, \mathrm{Q}=1.5-2.67$, $\mathrm{Qm}=1.99$, ellipsoid to subcylindrical, thinwalled, hyaline, smooth, strongly amyloid, with oil guttules. Basidia 14-19 × 6.5-9.5 $\mu \mathrm{m}$, clavate, bearing 4 sterigmata up to $3.5 \mu \mathrm{m}$ long. Lamella-edge homogeneous. Cheilocystidia $14-30 \times 5.5-10 \mu \mathrm{m}$, vesiculose, smooth, thinwalled, hyaline, with rostrate or acute or acuminate apex, often with an apical protrusion (4.5-15 $\times 1-2.5 \mu \mathrm{m})$ that may rarely show some branching. Pleurocystidia none. Lamellar trama regular to subregular; hyphae 3.5-17(29) $\mu \mathrm{m}$ wide, thin-walled, hyaline, faintly vinoid in Melzer's reagent. Subhymenium ramose. Pileus trama subregular; hyphae 6-30 $\mu \mathrm{m}$ wide, thin-walled, hyaline to pale greyish, faintly vinoid in Melzer's reagent. Pileipellis an epicutis; hyphae 2-11 $\mu \mathrm{m}$ wide, thin-walled, hyaline, with numerous lateral thorn-like protrusions (2.5-5 $\mu \mathrm{m}$ long), embedded in a gelatinous matrix, with prominent, loop-like clamp connections. Pileocystidia 23-45.5 $\times$ 10-16 $\mu \mathrm{m}$, elongated-clavate, thin-walled, with simple, conic or cylindrical excrescences $(0.5-$ $6 \times 0.5-1 \mu \mathrm{m})$. Detersile elements over the pileus of primordium $17-35.5 \times 14-25 \mu \mathrm{m}$, clavate or subglobose, thick-walled (up to 3.5 $\mu \mathrm{m})$ towards apex, hyaline, with simple, conic or cylindrical or coarse excrescences $(0.5-7 \times$ $0.5-2 \mu \mathrm{m})$. Pileus marginal cells $17-60 \times 9.5-$ $19 \mu \mathrm{m}$, clavate or lageniform, seldom with hairy protrusion (29.5-118 $\mu \mathrm{m}$ long), with simple, conic or cylindrical excrescences at the middle or towards apex $(0.5-6 \times 0.5-1 \mu \mathrm{m})$. Stipitipellis a cutis of smooth hyphae; hyphae 1.5-8 $\mu \mathrm{m}$ wide, thin-walled, hyaline. Caulocystidia 25-73.5 × 10.5-16 $\mu \mathrm{m}$, narrowly conic or narrowly lageniform, smooth, slightly thick-walled (up to $1.5 \mu \mathrm{m}$ ), often with a long protrusion (53-92.5 $\mu \mathrm{m}$ long). Stipe basal cells 13-93.5 × 5-20 $\mu \mathrm{m}$, clavate, vesiculose or utriform or cylindrical, thin-walled, hyaline. Stipe trama faintly vinoid in Melzer's reagent. Clamp connections seen on all hyphae.

On decaying woody shells of fruits of Vateria indica L. and on decaying leaves of various plants, in groups or scattered, JuneNovember.

Known distribution - widespread in Kerala State, India.

Material examined - India, Kerala State, Kozhikode District, Beypore: 22 Sept 2009, D.M. Aravindakshan DM334a (K(M) 178332); 3 Oct 2009, D.M. Aravindakshan DM334c (K(M) 178331, Holotype); 17 Aug 2011, D.M. Aravindakshan DM334g (K(M) 178333); Wayanad District, Thirunelli: 27 Nov 2009, D.M. Aravindakshan DM365 (K(M) 178334).

\section{Molecular analysis}

The ITS sequence-based molecular phylogenetic analyses generated trees with good support for most of the clades (Fig. 12). Topologies were similar in both maximum parsimony (MP) and maximum likelihood (ML) analyses. In Bayesian analysis, two independent runs reached a standard deviation of split frequencies of 0.01 after 40000 generations. Log-likelihood scores reached stationarity after 10000 generations. In both 


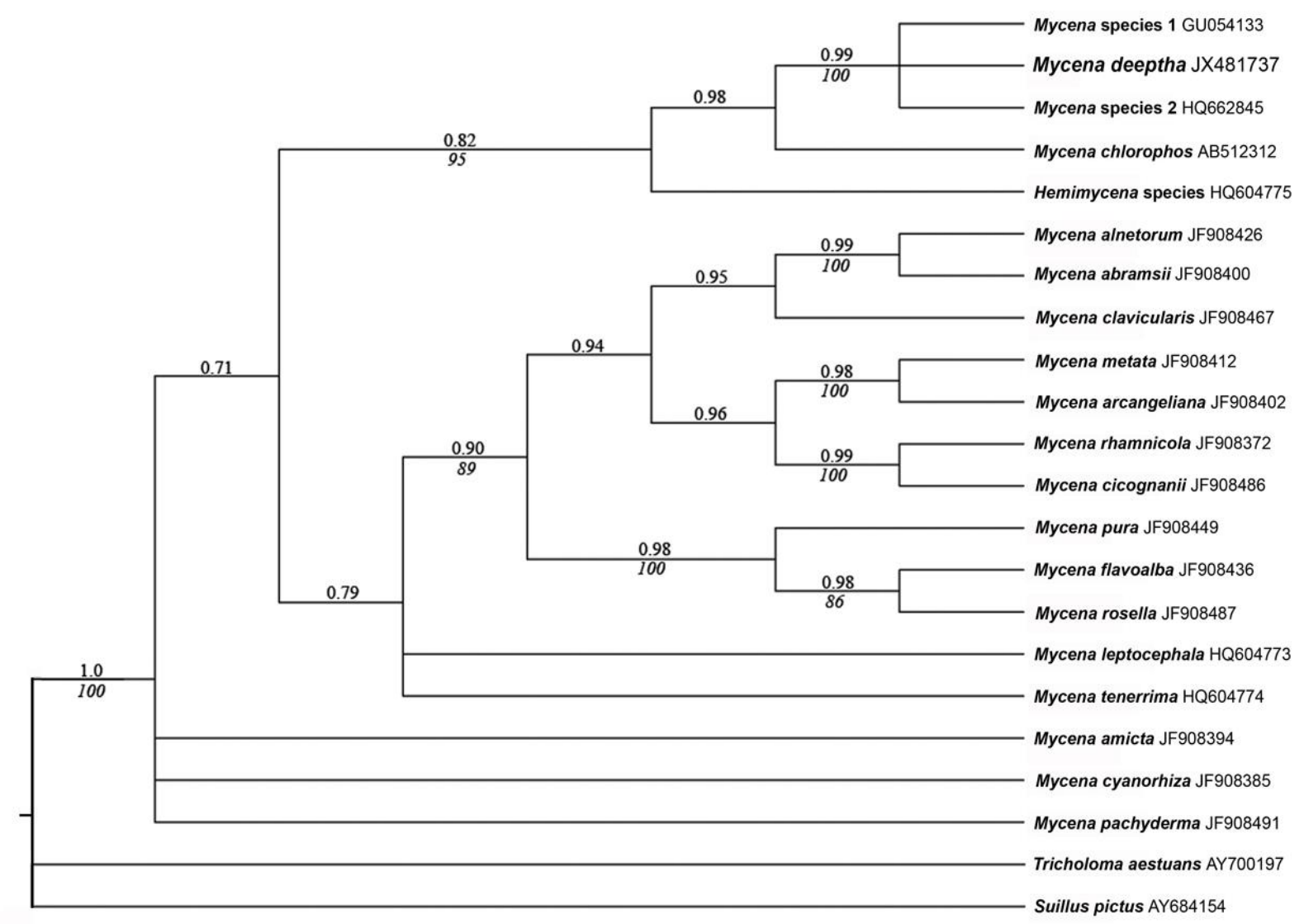

Fig 12 - Bayesian consensus tree obtained from Bayesian MCMC analysis using nuclear ITS sequence data. Values above the branches indicate Bayesian posterior probabilities greater than 0.70 and values below branches indicate Maximum Parsimony bootstrap values greater than $70 \%$. GenBank accession numbers are given after the taxonomic names.

MP and ML analyses, Mycena deeptha settled close to two unidentified Mycena species (GenBank accession numbers GU054133 and HQ662845, designated here as unidentified Mycena sp. 1 and unidentified Mycena sp. 2, respectively). This close relationship correlates with the result of the GenBank associated BLAST search. In BLAST search, the two unidentified species showed 99 and 100 percent sequence similarities, respectively. Mycena chlorophos another luminescent mushroom of the section Exornatae Maas Geest. settled close to the three Mycena species (Mycena deeptha, unidentified Mycena species 1 and 2). Mycena deeptha and the two unidentified Mycena species along with $M$. chlorophos always formed a distinct clade. This clade, although with weak bootstrap value, has good posterior probability support and can be identified as the section Exornatae clade.

\section{Discussion}

Characters such as a gelatinous pileipellis composed of hyphae that are covered with thorn-like excrescences, non-gelatinized stipitipellis, discoid stipe base and the luminescent mycelium of this species are hallmarks of section Exornatae. However, the combination of characters such as larger basidiomata, fimbriate pileal margin composed of cells with elongate protrusion, cheilocystidia and caulocystidia with elongate apical projections, and the presence of detersile elements over the primordium makes it unique and distinguishes it from other known members of that section (M. chlorophos, M. discobasis, M. chlorinosma and M. margarita).

Morphologically and microscopically, the present species is very similar to $M$. rhenana Maas Geest. \& Winterh. of sect. Basipedes (Fr.) Quel. that according to Desjardin et al. (2007) is best placed in sect. 
Exornatae. But that species has somewhat smaller basidiomata, numerous lamellae (2026), and marginal cells, caulocystidia, and stipe basal cells of a different type. In addition, it has no cheilocystidia and its pileipellis is not gelatinized. Also, that species is not known to be luminescent. Mycena cecidiophila A.P. Berg, Berg-Block, Noordel. \& Uljé placed under the monotypic section Cecidiophilae A.P. Berg, Berg-Block, Noordel. \& Uljé (van den Berg et al. 2000) is also found phenetically very similar to the present species. Remarkably, Noten \& Vannieuwerburgh (2009) and Aronsen (www.mycena.no/rhenana.htm) after reexamining the type of $M$. cecidiophila confirmed that it was conspecific with $M$. rhenana and they argued for the deletion of section Cecidiophilae.

Morphological characters such as larger basidiomata, fimbriate pileal margin composed of cells with elongate protrusion, cheilocystidia and caulocystidia with elongate apical projections, and the presence of detersile elements over the primordium when combined with results of the molecular analyses shows that Mycena deeptha can be placed in Mycena section Exornatae where it differs considerably from other related species including $M$. chlorophos. Remarkably, the ITS rDNA sequence of $M$. deeptha shows 99-100\% similarity with those of two unidentified Mycena species deposited in GenBank indicating that the new species may have a wider geographical distribution. Both the latter are environmental samples, the first (GenBank Acc. No. GU054133) from a soil sample collected from Korea and the second (GenBank Acc. No. HQ662845) from air filter samples with no locality details.

\section{Acknowledgements}

We thank the staff of the Photography Unit of the University of Calicut for their help in imaging the luminous mycelium of the new species.

\section{References}

Corner EJH. 1954. Further descriptions of luminous agarics. Transactions of the
British Mycological Society 37, 256271.

Desjardin DE, Capelari M, Stevani C. 2007. Bioluminescent Mycena species from São Paulo, Brazil. Mycologia 99, 317-331.

Desjardin DE, Oliveira AG, Stevani CV. 2008. Fungi bioluminescence revisited. Photochemical and Photobiological Sciences 7, 170-182.

Desjardin DE, Perry BA, Lodge DJ, Stevani C, Nagasawa E. 2010. Luminescent Mycena: new and noteworthy species. Mycologia 102, 459-477.

Felsenstein J. 1985. Confidence limits on phylogenies: An approach using the bootstrap. Evolution 39, 783-791.

Herring PJ. 1994. Luminous fungi. Mycologist 8, 181-183.

Horak E. 1978. Mycena rorida (Fr.) Quél. and related species from the southern hemisphere. Berichte der Schweizerischen Gesellschaft 88, 20-29.

Kornerup A, Wanscher JH. 1978. Methuen handbook of colour, 3rd edition. Eyre Methuen, London.

Maas Geesteranus RA. 1992. Mycenas of the Northern Hemisphere II. Conspectus of the Mycenas of the Northern Hemisphere. North-Holland, Amsterdam.

Noten L, Vannieuwerburgh L. 2009. Mycena rhenana versus Mycena cecidiophila. Sterbeeckia 28, 32-34.

Ronquist F, Huelsenbeck JP. 2003. MrBayes 3: Bayesian phylogenetic inference under mixed models. Bioinformatics 19, 15721574.

Swofford DL. 2002. PAUP*: phylogenetic analysis using parsimony (* and other methods), version 4. Sinauer, Sunderland, Massachusetts, USA.

van den Berg AP, van den Berg-Blok AE, Noordeloos ME, Uljé CB. 2000. A new species and a new section of the genus Mycena from the Netherlands. Persoonia $17(3), 48-485$.

Vydryakova GA, Gusev AA. Medvedeva SE. 2011. Effect of organic toxic compounds on luminescence of luminous fungi. Prikladnaya Biokhimiya i Mikrobiologiya $47,324-329$. 